\title{
Analysis of cladding-mode couplings for a lensed fiber integrated with a long-period fiber grating by use of the beam-propagation method
}

\author{
Wen-Yueh Su, Gia-Wei Chern, and Lon A. Wang
}

\begin{abstract}
The beam-propagation method (BPM) is employed to analyze the coupling behavior of our scheme proposed previously, which combines a lensed fiber and a long-period fiber grating (LPFG) [Chen and Wang, Appl. Opt. 39, 4490-4500 (2000)]. The influences of a core within the fiber lens are investigated. As for the fiber dependence of our coupling scheme, two typical fibers are studied: dispersion-shifted and single-mode, step-index fibers. With the BPM, the optimal coupling efficiencies for various source waists with corresponding lens radii and working distances are determined. We also compare the results with those obtained by use of the $A B C D$ method and found that the BPM gives better agreement with experimental results. (C) 2002 Optical Society of America

OCIS codes: $\quad 060.2310,050.2770,230.7370$.
\end{abstract}

\section{Introduction}

Efficient coupling of light from a laser diode (LD) to a single-mode fiber (SMF) plays an important role in optical fiber communication systems. The utilization of a lensed fiber provides desirable features such as being compact, stable, economical, and scalable to array-to-array coupling. ${ }^{1-6}$ However, optical feedbacks from the lensed fiber end face severely affect the operation of an LD, e.g., output power, dynamic properties, and emission spectra, and it has been shown that a long working distance between $\mathrm{LD}$ and fiber can reduce the effective reflectivity. ${ }^{7}$ Coupling schemes with a long working distance and that use an expanded-core fiber ${ }^{8}$ or a graded-index fiber were proposed.9,10 Recently, Chen and Wang'11,12 have demonstrated a new coupling method to obtain long working distances by utilizing a lensed fiber integrated with a long-period fiber grating (LPFG). In the scheme proposed here, light from an LD excites the fiber cladding mode that is then transferred into a fundamental core mode by the LPFG. Instead of coupling by the core mode, the cladding-mode field

The authors are with the Department of Electrical Engineering and Institute of Electro-Optical Engineering, National Taiwan University, Taipei, Taiwan. L. A. Wang's e-mail address is lon@ccms.ntu.edu.tw.

Received 3 April 2002.

0003-6935/02/316576-09\$15.00/0

(C) 2002 Optical Society of America has a larger numerical aperture that enables efficient coupling at a long working distance.

An LPFG is an important all-fiber band rejection filter. ${ }^{13}$ Resonant power transfer between core and cladding modes takes place when the phasematching condition is satisfied. With a properly chosen grating period, resonant coupling at a given wavelength between core and specific cladding mode can be realized. Fiber cladding modes are confined by the cladding-air interface, and the corresponding mode fields extend over the cladding region. ${ }^{14} \mathrm{Be}-$ cause of the larger cladding-mode radii, it is possible to realize couplings with long working distances by launching light into the cladding modes. Since the fiber cladding modes are linearly polarized $^{14}$ and have real-valued field distributions over the transverse plane, to have the larger excitation efficiency it is necessary to introduce a lensed fiber to compensate the curvature of the incident beam. With the properly chosen radii of the fiber lenses, coupling efficiencies of $78 \%$ and $35 \%$ at working distances 250 and 110 $\mu \mathrm{m}$ were demonstrated for fiber-to-fiber and LD-tofiber couplings, respectively. ${ }^{12}$

Although the properties of cladding-mode couplings can be analyzed with the $A B C D$ ray optics model, ${ }^{12}$ the effects of fiber core cannot be properly taken into account. In this research, we use the beam-propagation method (BPM) ${ }^{15,16}$ to investigate the field evolution through the lensed fiber with and without a core. The BPM is a powerful technique for analyzing the wave propagation characteristics in a 
wide variety of inhomogeneous media, ${ }^{17-19}$ including the design of lensed fiber coupling. ${ }^{10,16}$ Through the BPM analysis, we examine the transformation of the electric field when the incident beam propagates into the fiber lens. The dependence of mode excitation efficiencies on fiber cores is also studied.

This paper is organized as follows. In Section 2, the proposed scheme is reviewed, and the $A B C D$ matrix method is outlined. We highlight the BPM to be employed for the analysis of the lensed fiber. A mode-expansion method is then used to calculate the coupling efficiency. In Section 3, the behavior of cladding-mode coupling is found to depend significantly on the size of the initial waist. For two exemplary initial waists, we compare the results among those obtained by the $A B C D$ method, the BPM, and the measured ones. We also discuss their deviations from one another. The fiber dependence of the proposed coupling scheme and the limitation of the waist of the light source used for coupling are also studied. Section 4 concludes this study.

\section{Two Analysis Methods}

The working principle of the LPFG-assisted fiber coupling has been reported previously. ${ }^{11,12}$ In the scheme proposed here, the first cladding mode of an $\mathrm{SMF}$ is to be optimized to have the high excitation efficiency for an incident Gaussian beam. The excited cladding mode is then coupled to the fundamental core mode by an LPFG. To enhance the excitation efficiency, the fiber end should be properly shaped to compensate the curvature of the incident beam. A simple lensed fiber is used for this purpose. ${ }^{11}$ Although the use of $A B C D$ matrix method is straightforward and leads to a qualitative agreement with the experimental results for calculating coupling efficiency, ${ }^{12}$ the effects of the core region, the actual shape of fiber lens, and the radiation loss cannot be properly considered. In the following, we shall use the BPM to compare field evolution and mode excitation of a fiber lens with and without a fiber core. A mode-expansion method is then used to calculate the excitation efficiency of the corresponding modes after propagation through the lens.

\section{A. Analytic Approach Based on the $A B C D$ Method}

A schematic coupling diagram is shown in Fig. 1. A laser source in plane $Z=Z_{1}$ has the Gaussian beam parameters of waist $W_{1}=W_{0}$ and curvature $R_{1}=\infty$. After propagation through a working distance $Z_{s}$, the beam parameters become

$$
\begin{aligned}
& W_{2}=W_{0}\left[1+\left(\frac{Z_{s}}{Z_{0}}\right)^{2}\right]^{1 / 2}, \\
& R_{2}=Z_{s}\left[1+\left(\frac{Z_{0}}{Z_{s}}\right)^{2}\right],
\end{aligned}
$$

where $Z_{0}=n_{1} \pi W_{0}^{2} / \lambda_{0}$ is the Rayleigh parameter; $n_{1}$ is the refractive index in region I; and $\lambda_{0}$ is the operating wavelength. $W_{2}$ and $R_{2}$ are the new parameters in plane $Z_{2}$, i.e., the front end plane of the fiber

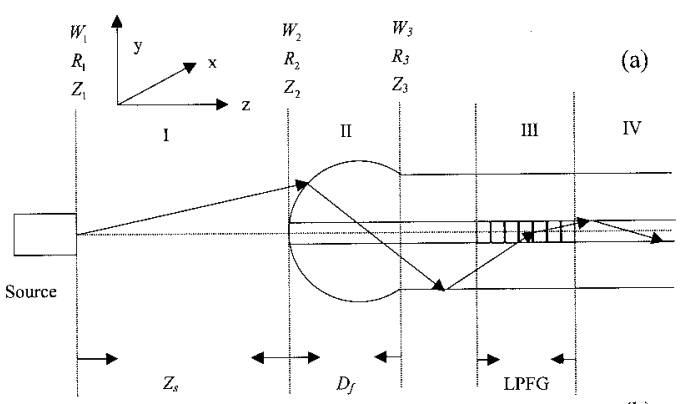

(a)

(b)

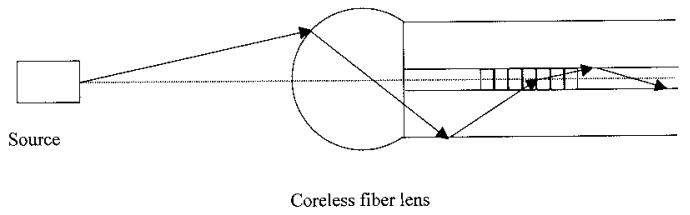

Fig. 1. Schematic diagrams of the coupling scheme studied in the text for (a) a cored fiber lens and (b) a coreless fiber lens integrated with an LPFG. Abbreviations are defined throughout the text.

lens. At normal incidence, the composite $A B C D$ matrix after the beam propagating through the lens and a free distance $D_{f}$ is

$$
\begin{aligned}
\mathbf{M} & =\left[\begin{array}{ll}
A & B \\
C & D
\end{array}\right], \\
& =\left[\begin{array}{cc}
1+D_{f} \frac{n_{1}-n_{2}}{n_{2} R_{f}} & D_{f} \frac{n_{1}}{n_{2}} \\
\frac{n_{1}-n_{2}}{n_{2} R_{f}} & \frac{n_{1}}{n_{2}}
\end{array}\right],
\end{aligned}
$$

where $R_{f}$ is the radius of the lens and $n_{2}$ is the refractive index in region II. The transformed beam parameters in plane $Z_{3}$ before the LPFG then become

$$
\begin{aligned}
W_{3} & =W_{2}\left[\left(A+\frac{B}{R_{2}}\right)^{2}+\left(\frac{\lambda_{0} B}{\pi W_{2}^{2}}\right)^{2}\right]^{1 / 2}, \\
R_{3} & =\frac{\left(A+\frac{B}{R_{2}}\right)^{2}+\left(\frac{\lambda_{0} B}{\pi W_{2}^{2}}\right)^{2}}{\left(A+\frac{B}{R_{2}}\right)\left(C+\frac{D}{R_{2}}\right)+B D\left(\frac{\lambda_{0}}{\pi W_{2}^{2}}\right)^{2}} .
\end{aligned}
$$

Assume that the incident Gaussian beam is linearly polarized and that the normalized electric field in plane $Z=Z_{3}$ can be expressed

$$
\mathbf{E}_{3} \cong \hat{\mathbf{y}} E_{3, y}=\hat{\mathbf{y}}\left(\frac{1}{\pi W_{3}^{2}}\right)^{1 / 2} \exp \left(-\frac{r^{2}}{W_{3}^{2}}-\frac{i k_{1} r^{2}}{2 R_{3}}\right),
$$

where $r=\left(x^{2}+y^{2}\right)^{1 / 2}$ is the radial distance and $k_{1}=$ $n_{2} 2 \pi / \lambda_{0}$. This field will be used in the following mode-expansion analysis for computing the mode excitation efficiency.

B. Numerical Approach Based on the Wide-Angle Beam Propagation Method

Here we apply the BPM to investigating field evolution in the fiber lens so that the effects of the finite 
cladding radius and the existence of fiber core can be properly taken into account. Because both the core and the cladding modes under consideration are almost linearly polarized, ${ }^{14}$ the electrical field with azimuthal order $l$ can be expressed

$$
\mathbf{E}(r, \phi, z)=\hat{\mathbf{y}} \psi(r, z) \exp \left(-i k n_{a} z\right) \exp (i l \phi),
$$

where $n_{a}$ is the properly chosen reference refractive index and $k$ is the wave number in free space. In the following, we consider a circularly symmetric guiding structure, i.e., $n=n(r, z)$. The scalar field $\psi$ satisfies the following wave equation

$$
\begin{aligned}
2 i k n_{a} \frac{\partial \psi}{\partial z}-\frac{\partial^{2} \psi}{\partial z^{2}} & =\frac{\partial^{2} \psi}{\partial r^{2}}+\frac{1}{r} \frac{\partial \psi}{\partial r}-\frac{l^{2}}{r^{2}}+k^{2}\left(n^{2}-n_{a}^{2}\right) \psi \\
& =\mathbf{H} \psi,
\end{aligned}
$$

where $\mathbf{H}$ is the corresponding finite-difference matrix. The corresponding matrix elements can be found in, e.g., Ref. 17 A $(1,1)$ Padé approximation is used to derive the following equation ${ }^{19}$ :

$$
\frac{\partial \psi}{\partial z}=\frac{-i\left(\mathbf{H} / 2 k n_{a}\right)}{1+\left(\mathbf{H} / 4 k^{2} n_{a}^{2}\right)} \psi .
$$

By discretizing the nonuniform guiding structure into a stepwise one and using the finite-difference scheme, one can integrate Eq. (9) to give

$$
\begin{aligned}
\left(1+\frac{1+i k n_{a} \Delta z}{4 k^{2} n_{a}{ }^{2}} \mathbf{H}\right) & \psi(z+\Delta z)= \\
& \left(1+\frac{1-i k n_{a} \Delta z}{4 k^{2} n_{a}{ }^{2}} \mathbf{H}\right) \psi(z) .
\end{aligned}
$$

With the initial condition $\psi\left(z=Z_{2}\right)=E_{2}$, the field evolution through the fiber lens can thus be obtained. Since the field under consideration has a wide extension along the radial distance, to prevent artificial reflections from the window boundary, it is necessary to employ a transparent boundary condition. ${ }^{20}$

Again, let $\mathbf{E}_{3}$ be the field after propagating through the lens and the mode-expansion method be used to calculate the excitation efficiency. The guided modes with circular symmetry are the $\mathrm{LP}_{0 m}$ modes, with $m=1$ corresponding to the core mode and $m>$ 1 corresponding to cladding modes. Let $\mathbf{e}_{0 m}$ be the $\mathrm{LP}_{0 m}$ mode field that is normalized to carry $1-\mathrm{W}$ optical power. With the orthogonality relation, the field $\mathbf{E}_{3}$ can be expanded as

$$
\mathbf{E}_{3}=\sum_{m} \mathbf{E}_{0 m}+\mathbf{E}_{\mathrm{rad}}=\sum_{m} a_{m} \mathbf{e}_{0 m}+\mathbf{E}_{\mathrm{rad}},
$$

where $\mathbf{E}_{0 m}$ is the field projection in mode $\mathrm{LP}_{0 m}$ and $\mathbf{E}_{\text {rad }}$ represents the unconfined radiation modes. The excitation efficiency of mode $\mathrm{LP}_{0 m}$ is the ratio of power carried by this mode to the incident power. By normalizing the incident field $\mathbf{E}_{0}$ to carry power 1
$\mathrm{W}$, the overall excitation efficiency of mode $\mathrm{LP}_{0 m}$ is then given by

$$
\eta_{0 m}=\left|\iint \mathbf{E}_{3} \cdot \mathbf{e}_{0 m}^{*} \mathrm{~d} A\right|^{2}=\left|a_{m}\right|^{2} .
$$

The total coupling efficiency at wavelength $\lambda$ can thus be expressed with the excitation efficiencies and the transmission of the LPFG as

$$
\frac{\int\left[\eta_{01}(\lambda) T_{01}(\lambda)+\sum_{m=2,3, \ldots} \eta_{0 m}(\lambda) T_{0 m \rightarrow 01}(\lambda)\right] P(\lambda) \mathrm{d} \lambda}{\int P(\lambda) \mathrm{d} \lambda},
$$

where $T_{01}(\lambda)$ is the self-transmission of core mode through LPFG at wavelength $\lambda$ and $T_{0 m \rightarrow 01}(\lambda)$ for $m=2,3, \ldots$ is the cross transmission of LPFG from cladding mode $\mathrm{LP}_{0 m}$ to core mode at wavelength $\lambda$. $P(\lambda)$ is the spectral power distribution of source. For a practical design, the grating period is adjusted to obtain the resonance between the fundamental core mode $\left(\mathrm{LP}_{01}\right)$ and the first cladding mode $\left(\mathrm{LP}_{02}\right)$ at the source wavelength. Assume the source beam is monochromatic at $\lambda_{0}$, then the total coupling efficiency can be approximated by

$$
\eta_{\text {total }} \cong\left[1-L\left(\lambda_{0}\right)\right] \eta_{01}{ }^{\mathrm{co}}+L\left(\lambda_{0}\right) \eta_{02}{ }^{\mathrm{cl}},
$$

where $L\left(\lambda_{0}\right)$ is the loss of LPFG at $\lambda_{0}$, i.e., $L \cong 1-$ $T_{01}=T_{02 \rightarrow 01}$. Here we assume the resonance between the core and the first cladding modes is ideal, i.e., the energy of the first cladding mode can be completely transferred into the core mode by an LPFG. From Eq. (14), it can be seen that to have a large coupling efficiency, $L\left(\lambda_{0}\right)$ should be equal to 1 , and the total efficiency is the excitation efficiency of the first cladding mode.

\section{Results and Discussion}

By using the BPM, one can study in detail the transformation of the electric field within the fiber lens. In the following, therefore, we shall first compare the field evolution within a fiber lens with and without a core. Specifically, we shall use the BPM to investigate the influences of the existence of a core on mode excitations and coupling efficiencies. Then we shall study the effects of a core region on the coupling behavior. For the fiber dependence of our coupling scheme, two typical fibers are considered, one is the standard SMF with step-index (SI) profile, and the other is a dispersion-shifted fiber (DSF), as used in our experiment. We also study the dependence of coupling efficiency on the initial waist of source. The results with the BPM are compared with those from the $A B C D$ method and from the experiment reported previously.11,12 Note that two kinds of light sources are used. The first is for fiber-to-fiber cou- 


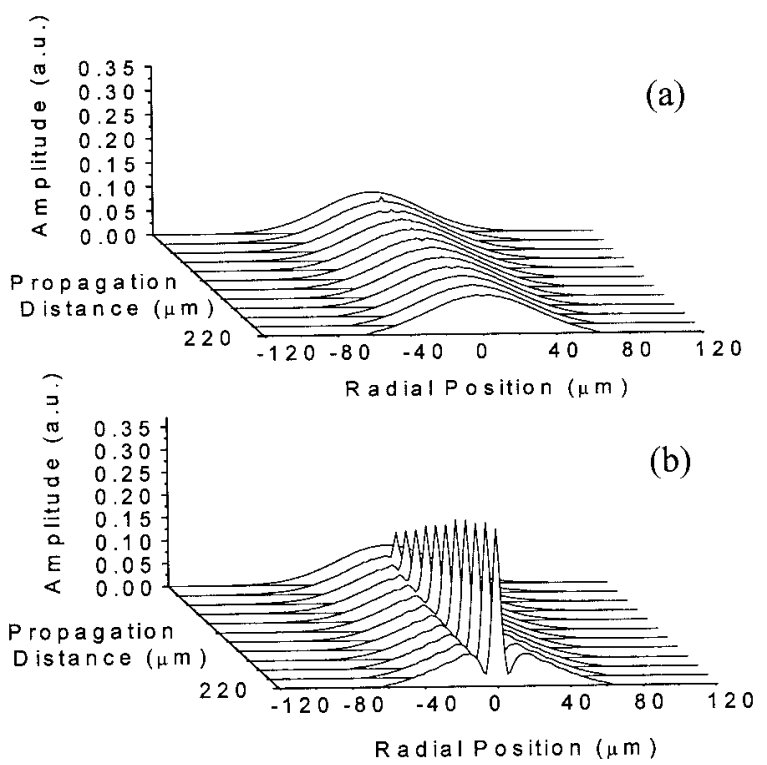

Fig. 2. Evolution of a Gaussian beam through (a) the coreless lens and (b) the cored lens from the source beam with an initial waist of $3.25 \mu \mathrm{m}$ and at a working distance of $250 \mu \mathrm{m}$.

pling, which resembles a source with a large waist. The second is for LD-to-fiber coupling in which the source is Fabry-Perot laser diode.

\section{A. Analysis of the Coupling Scheme}

Two types of fiber lens structures with and without a core, as shown in Fig. 1, are considered. The source wavelength is $1.56 \mu \mathrm{m}$. To study the effects of the coupling behavior on the beam waists, we consider two beam waists 3.25 and $1 \mu \mathrm{m}$, exemplifying large and small waists, respectively. The radii of the fiber lens are $R_{f}=120 \mu \mathrm{m}$ for coupling the 3.25- $\mu \mathrm{m}$-waist source and $R_{f}=75 \mu \mathrm{m}$ for the $1-\mu \mathrm{m}$-waist source. The numerical window size in the BPM simulation is $300 \mu \mathrm{m}$, and the number of grids is 1000 , corresponding to a $\Delta r=0.3 \mu \mathrm{m}$. The step size $(\Delta z)$ ranges from 0.025 to $0.2 \mu \mathrm{m}$ from the consideration of required accuracy and numerical stability. The evolution of the field amplitude through the fiber lens is shown in Fig. 2 for the coupling of the $3.25-\mu \mathrm{m}$-waist source. The working distance $Z_{\mathrm{s}}$ is $250 \mu \mathrm{m}$, and the step size is chosen to be $\Delta z=0.025 \mu \mathrm{m}$. Figure 2(a) shows the transformation of an incident Gaussian beam in a coreless lens, whereas Fig. 2(b) corresponds to a cored DSF lens. Since the divergence angle of the Gaussian beam from the source is small $\left(\sim 8.75^{\circ}\right.$ in halfangle), there is not much beam truncation owing to the finite lens size. For the coreless case as shown in Fig. 2(a), the transformation of the incident Gaussian beam should be close to that simulated by use of the $A B C D$ method, which will be illustrated [see Fig. 5(a) below]. Because of the small amplitude at the cladding boundaries, the reflection from the cladding-air interface is not significant. However, the multiple reflection of field at the core-cladding interface induces a central peak in the core region as shown in Fig. 2(b). At the end of the cored-fiber lens, the field

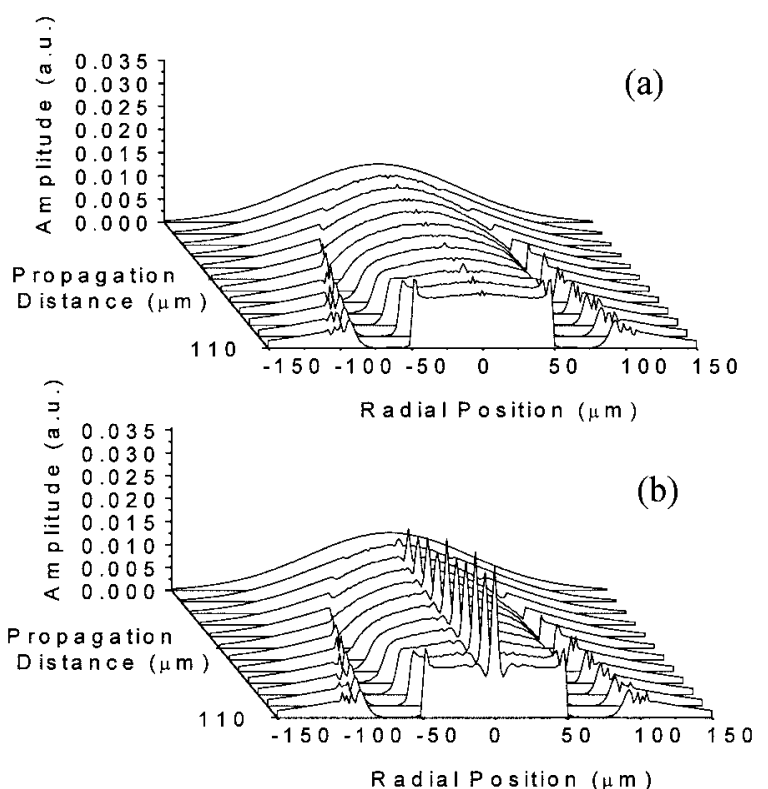

Fig. 3. Evolution of a Gaussian beam through (a) the coreless lens and (b) the cored lens from the source beam with an initial waist of $1 \mu \mathrm{m}$ and at a working distance of $160 \mu \mathrm{m}$.

amplitude has a narrow peak in the central part and a dip at $r= \pm 6.6 \mu \mathrm{m}$. By projecting the transformed field in the various guided modes, the field is found to be composed mainly of modes $\sim 8.6 \% \mathrm{LP}_{01}$ and $\sim 83 \%$ $\mathrm{LP}_{02}$.

Figure 3 shows the corresponding field evolution through a fiber lens for the coupling of the smallwaist source. The coreless case is shown in Fig. 3(a), whereas the case with a DSF core is shown in Fig. 3(b). The working distance $Z_{s}$ is $160 \mu \mathrm{m}$ and the step size is set the same as that in Fig. 2. Since the divergence angle of the source is $\sim 28.45^{\circ}$ in halfangle and the incident beam size is large compared with the lens diameter, the amplitude of electric field at the cladding-air interface is still large and will be reflected. It can be seen from Fig. 3 that the incident Gaussian beam is truncated by the lens boundary and that partial energy is reflected from the cladding-air interface. The reflected field then interferes with the outgoing components. This interference causes an oscillation in the dependence of coupling efficiency on the working distance [see Fig. 5(b) below]. In Fig. 3(b), an extra peak results in the central region owing to the existence of a DSF core.

Since the coupling efficiency depends on the excitation efficiencies of the core $\left(\mathrm{LP}_{01}\right)$ and the first cladding $\left(\mathrm{LP}_{02}\right)$ modes, it would be useful to study the evolution of fraction of power in local guiding modes $\left(\mathrm{LP}_{01}\right.$ and $\left.\mathrm{LP}_{02}\right)$ of the lens with a DSF core. The local guiding structure has a cladding radius defined by $R(z)=\left[R_{f}^{2}-\left(R_{f}-z\right)^{2}\right]^{1 / 2}$, and the local modes are solved from the corresponding local guiding structure by use of the finite-difference method. In Fig. 4, we show the evolution of coupled power in local modes for the coupling from 3.25- and 1- $\mu \mathrm{m}$-waist sources. In both cases, the fraction of power in the local core 


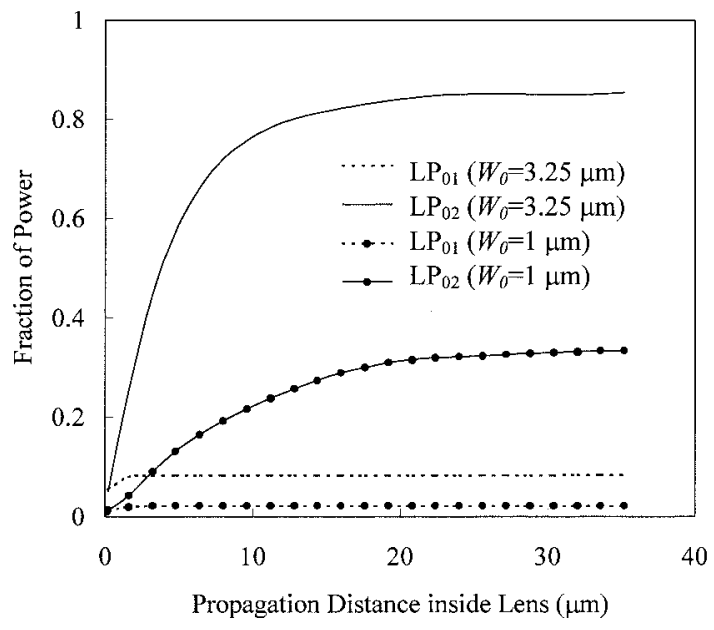

Fig. 4. Fraction of power in local modes $\mathrm{LP}_{01}$ and $\mathrm{LP}_{02}$ as a function of the propagation distance inside lens. The zero point of the propagation distance corresponds to the entrance of lens.

mode is small (less than 0.1) and remains nearly a constant as the field propagates inside the lens. On the other hand, the fraction of power in local cladding mode increases monotonically and saturates at distances greater than $30 \mu \mathrm{m}$. The coupled fractional power in the local mode $\mathrm{LP}_{02}$ saturates at $\sim 83 \%$ for coupling the $3.25-\mu \mathrm{m}$-waist and $\sim 31 \%$ for the $1-\mu \mathrm{m}$ waist sources.

The calculated coupling efficiency versus the working distance for couplings from a 3.25- $\mu \mathrm{m}$-waist source, obtained with Eq. (14), is shown in Fig. 5(a). When an ideal transmission LPFG is used, the coupling efficiency is equal to the excitation efficiency of the cladding mode $\mathrm{LP}_{02}$. Three curves are shown in Fig. 5(a), corresponding to the results simulated with and without a fiber core by the BPM and by the $A B C D$ method. The maximum coupling efficiencies are found to be approximately $83 \%, 84 \%$, and $86 \%$ at $Z_{\mathrm{s}}=$ $250 \mu \mathrm{m}$. The estimated corresponding longitudinal tolerances at a 1-dB loss are found to be approximately $60 \mu \mathrm{m}$ for all the three cases. Similar coupling behaviors for a lensed fiber with and without a core indicates that the proposed scheme does not depend on the existence of a core. Although the field evolution is significantly influenced by the existence of the fiber core, as shown in Figs. 2 and 3, the cladding-mode excitation efficiency is insensitive to the existence of the core. This can be understood as follows. Let $E_{y}^{(a)}$ and $E_{y}^{(b)}$ be the transformed electric fields for the lens structure with and without the core, respectively, in plane $Z_{3}$ with the same input field. For example, $E_{y}^{(a)}$ and $E_{y}^{(b)}$ are fields in Figs. 2(a) and 2(b) at the propagation distance of $220 \mu \mathrm{m}$. The extra field due to the presence of core is the difference of these two fields, i.e., $E_{\text {diff }}=E_{y}^{(b)}-E_{y}^{(a)}$. In Fig. 6, we show the amplitude of the difference field $E_{\text {diff }}$, and the phase is shown in the inset. The projections of this difference field into $\mathrm{LP}_{01}$ and $\mathrm{LP}_{02}$ modes are 0.96 and 0.0007 , respectively. It means that the difference field is quite similar to the core mode and

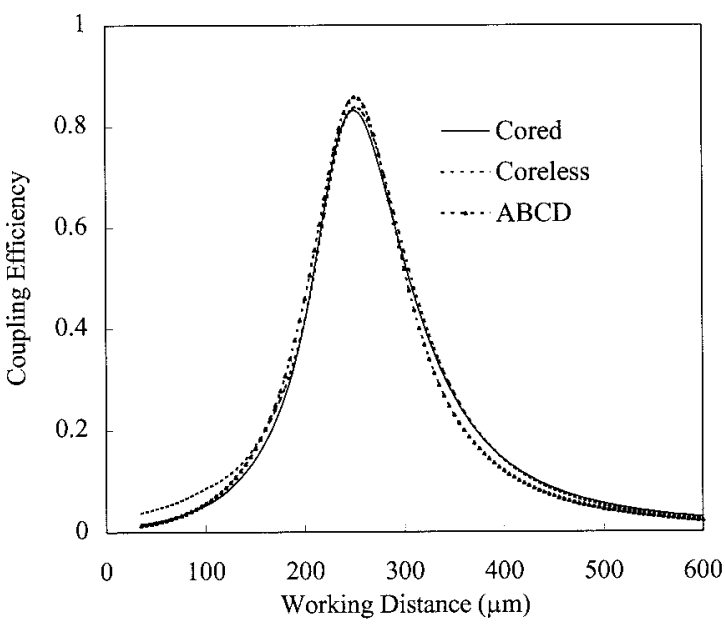

(a)

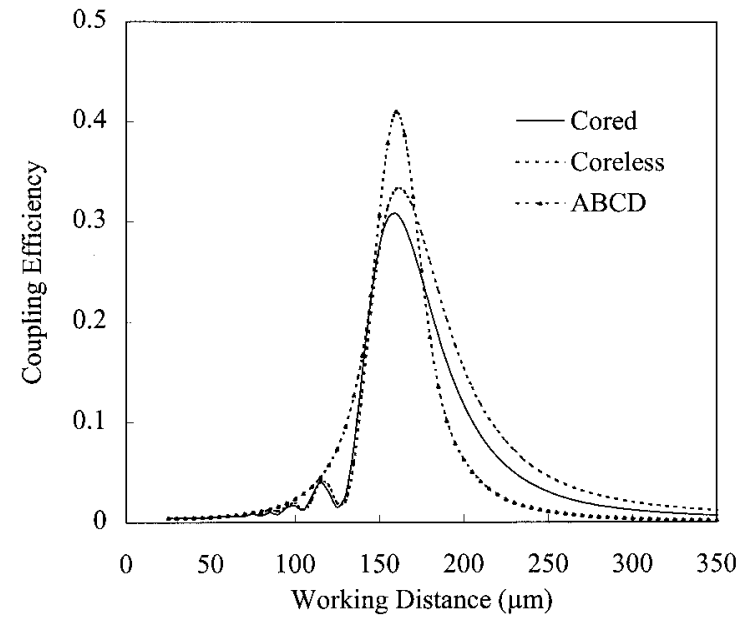

(b)

Fig. 5. Calculated coupling efficiencies versus the working distance by use of the BPM (cored and coreless lens) and by use of the $A B C D$ method for couplings from the source (a) with an initial waist of $3.25 \mu \mathrm{m}$ and (b) with an initial waist of $1 \mu \mathrm{m}$.

almost orthogonal to the first cladding mode. The small overlapping with the cladding mode $\mathrm{LP}_{02}$ indicates that the extra field induced by a core contributes negligibly to the coupling efficiency, because the proposed coupling scheme is dominated by the first cladding-mode excitations. The calculated coupling efficiency by the BPM is also close to the result of the $A B C D$ method. For the case of large-waist source, therefore, the coupling efficiency can well be simulated with the $A B C D$ method.

Figure 5(b) shows the coupling efficiencies versus the working distance for coupling a $1-\mu \mathrm{m}$-waist source. The maximum coupling efficiencies are found to be approximately $31 \%, 33 \%$, and $41 \%$ at $Z_{\mathrm{s}}=160 \mu \mathrm{m}$ for the cored-lens, the coreless lens (from the $\mathrm{BPM}$ ), and the $A B C D$ method, respectively, and the estimated corresponding longitudinal tolerances at a 1-dB loss are found to be approximately 30,35 , and $20 \mu \mathrm{m}$, respectively. For small-waist sources, the divergence angle of the beam is large. Significant reflection occurs at the 


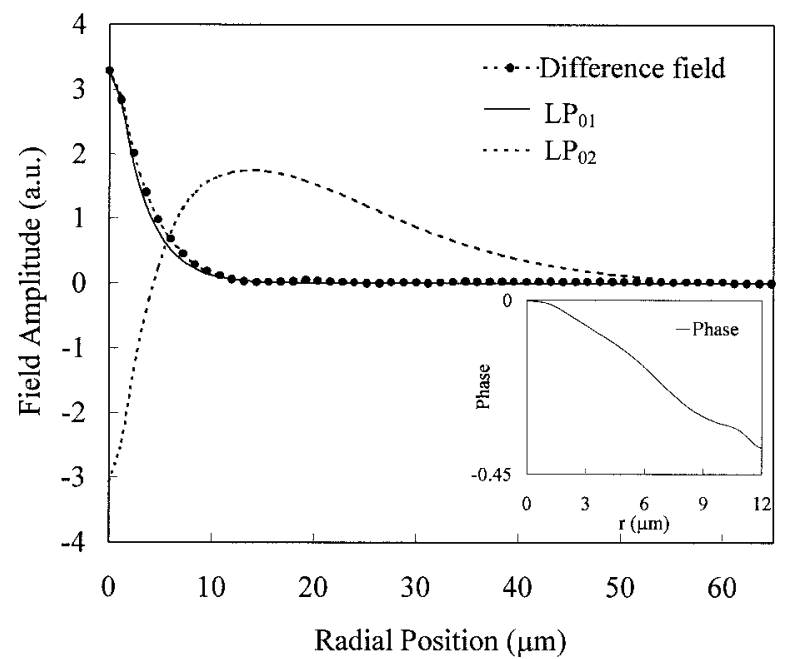

Fig. 6. Amplitude of the difference field $E_{\text {diff, }}$, which is defined in the text and also compared with the electric field of modes $\mathrm{LP}_{01}$ and $\mathrm{LP}_{02}$. The inset shows the phase of the difference field and the radial position is denoted as $r$.

cladding-air interface and manifests itself in an oscillating behavior in the coupling efficiency over the working distance owing to interference. Note that such an effect cannot be taken care of properly by use of the $A B C D$ method.

Figure 7(a) shows the calculated coupling efficiencies versus the working distances for fiber-to-fiber coupling, and the measured data taken from the previous work ${ }^{11,12}$ are included. The source beam waist is chosen to be $3.25 \mu \mathrm{m}$, corresponding to the beam waist of the core mode, and the lens radius is $120 \mu \mathrm{m}$. The maximum coupling efficiency is $\sim 78 \%$ at a working distance of $250 \mu \mathrm{m}$, and the coupling efficiency has a longitudinal tolerance of $\sim 60 \mu \mathrm{m}$ at a 1 -dB loss. The measured coupling efficiencies are larger than the calculated ones in the short working distance, which can be attributed to the reduced transmission loss of LPFG. The excited energy in $\mathrm{LP}_{01}$ mode is relatively large in short working distances. From Eq. (14), when $L\left(\lambda_{0}\right)$ is not equal to 1 , both core $\left(\mathrm{LP}_{01}\right)$ and first cladding $\left(\mathrm{LP}_{02}\right)$ modes contribute to the coupling efficiency. As a result, the measured coupling efficiencies are larger. The possible reasons for the reduced transmission loss are the wavelength mismatch and the induced bending that also causes the resonant peak of the LPFG to shift during the assembly of the LPFG. Figure 7(b) shows the calculated coupling efficiencies versus the working distances for LD-to-fiber coupling with a lens radius of $75 \mu \mathrm{m}$. The previous reported data are included for comparison. ${ }^{11,12}$ The maximum coupling efficiency is $\sim 26 \%$ at a working distance of $160 \mu \mathrm{m}$ for LD-to-fiber coupling, and the coupling efficiency has a longitudinal tolerance of $\sim 50 \mu \mathrm{m}$ at a $1-\mathrm{dB}$ loss. The LD's beam profile has an ellipticity of 2.5 and the near-field beam waists are estimated to be 0.71 and $1.78 \mu \mathrm{m}$ in the vertical and the parallel directions, respectively. For a general noncircularly symmetric input beam,

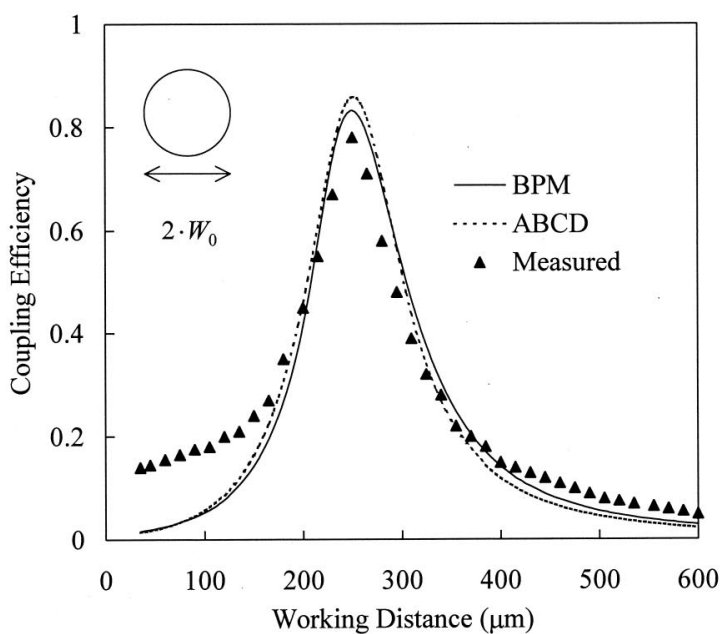

(a)

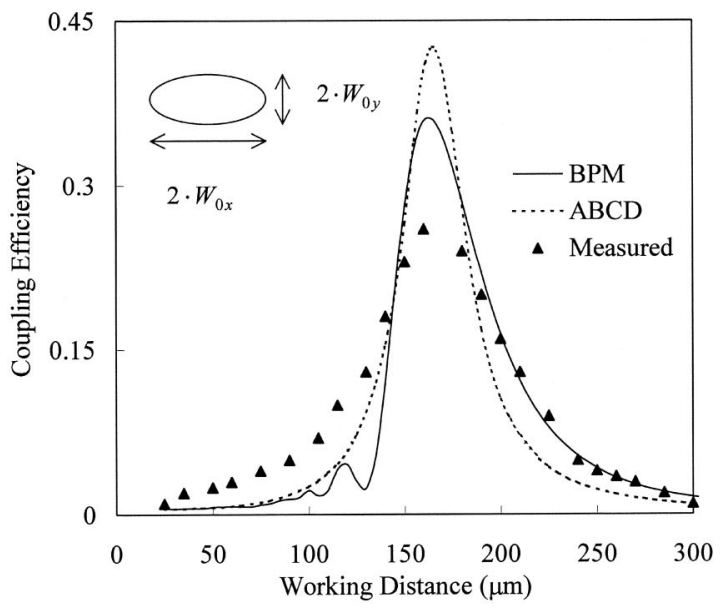

(b)

Fig. 7. Measured coupling efficiencies versus the working distance for (a) fiber-to-fiber coupling, where $W_{0}=3.25 \mu \mathrm{m}$ with a lens radius of $120 \mu \mathrm{m}$ and (b) LD-to-fiber coupling, where $W_{0 x}=$ $1.78 \mu \mathrm{m}$ and $W_{0 y}=0.71 \mu \mathrm{m}$ with a lens radius of $75 \mu \mathrm{m}$. The results are also compared with the calculated ones by use of the BPM and by use of the $A B C D$ method.

we may expand the field into summation of different azimuthal orders,

$$
\mathbf{E}=\sum_{l=0,1,2, \ldots} \mathbf{E}_{l} \exp (i l \phi),
$$

where the $l$ th component is given by the Fourier formula. Since the guiding structure of lensed fiber is circularly symmetric, it conserves the angular momentum of the incident beam. Consequently, each azimuthal order propagates independently. Because the cladding modes under consideration have $l=0$, higher-order components cannot excite these guided modes. Here, we use the BPM with $l=0$ to study the transformation of the zeroth-order component of the elliptical beam. But in the $A B C D$ method the elliptical source is dealt with directly. The calculated maximum coupling efficiencies are approximately $32 \%$ and $43 \%$ at $Z_{s}=165 \mu \mathrm{m}$ with the $\mathrm{BPM}$ and the $A B C D$ method, respectively, and the 


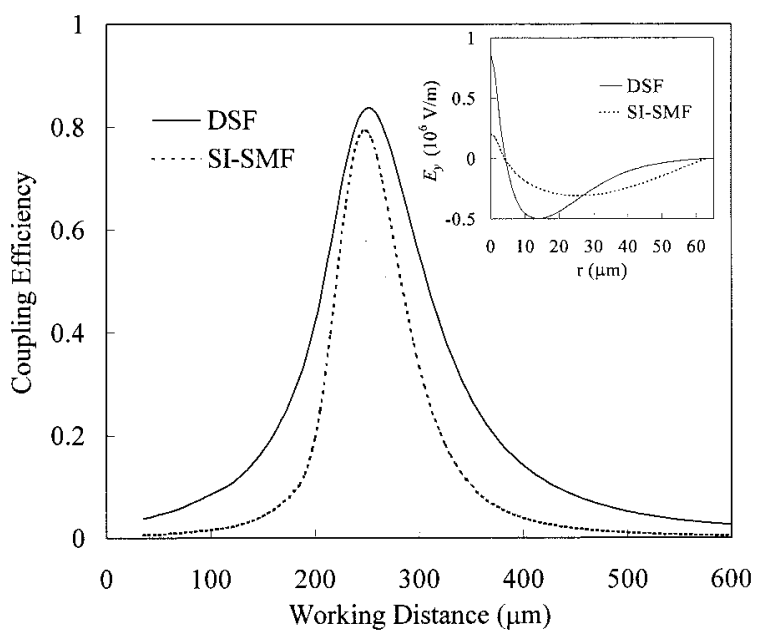

Fig. 8. Comparison of calculated coupling efficiencies of a DSF and an SI-SMF as a function of the working distance for couplings from a source with a $3.25-\mu \mathrm{m}$ waist. The inset shows the field distribution for the first cladding modes of a DSF and an SI-SMF.

estimated corresponding longitudinal tolerances are approximately 30 and $20 \mu \mathrm{m}$, respectively. The measured coupling efficiencies in the short working distance are larger than the calculated ones for the same reason as described above. With the use of the BPM, the oscillating behavior in the calculated coupling efficiency over the working distance is attributed to the effect of the lens boundary as proved previously.

\section{B. Fiber Dependence}

Two fibers are considered: SMF and DSF. As discussed previously, the transmission loss of LPFG should be equal to 1 for the best coupling. From the coupled-mode theory, the transmission loss at resonance wavelength can be expressed

$$
L=1-\cos ^{2}\left(\kappa_{\text {co-cl }} l_{g}\right),
$$

where $l_{g}$ is the length of LPFG and $\kappa_{\text {co-cl }}$ is the coupling constant that has the following form, ${ }^{14}$

$$
\kappa_{\text {co-cl }}=\frac{\omega \varepsilon_{0} \Delta n_{g}}{2} \int_{A_{\mathrm{co}}} n_{\mathrm{co}} \mathbf{E}_{02} \cdot \mathbf{E}_{01} * \mathrm{~d} A,
$$

where $n_{\text {co }}$ is the refractive index of the fiber core and $\Delta n_{g}$ is the index difference of the grating. Figure 8 shows the calculated coupling efficiencies versus the working distance for couplings from the $3.25-\mu \mathrm{m}$ waist source. The parameters used for calculation are the same as before. The inset shows the calculated electric field $E_{y}$ for the first cladding modes $\left(\mathrm{LP}_{02}\right)$ of a DSF and an SI-SMF. With the field distributions of modes $\mathrm{LP}_{01}$ and $\mathrm{LP}_{02}$, the coupling constants $\kappa_{\text {co-cl }}$ of the two kinds of fibers can be calculated with Eq. (17). If it is assumed that the photoinduced $\Delta n_{g}$ is the same for both fibers, the coupling constant of DSF is $\sim 3.6$ times that of a SI fiber, which explains why the transmission loss of the first cladding mode of an SI-SMF is relatively small.

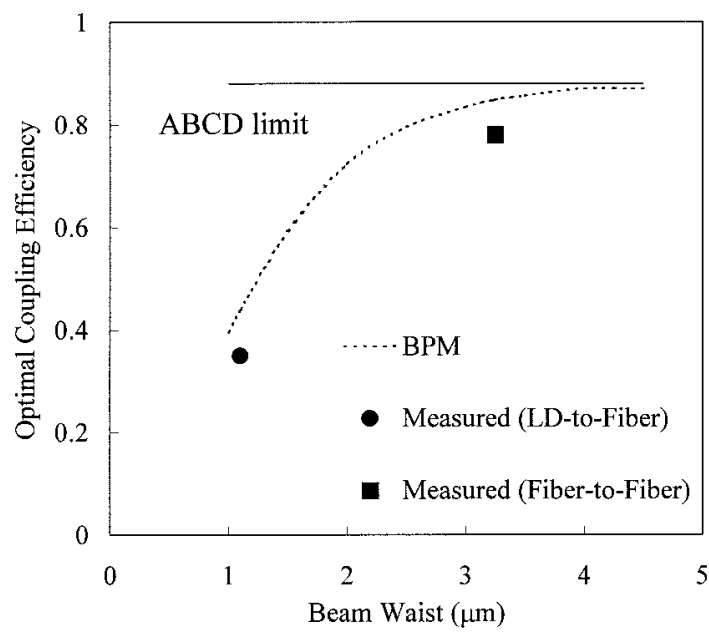

Fig. 9. Calculated and measured optimal coupling efficiency versus beam waist.

Assuming the same coupling constant, we compare the calculated coupling efficiency of a DSF to that of an SI-index SMF. The optimal working distance for the maximum coupling efficiency is at $250 \mu \mathrm{m}$, and the coupling efficiencies of a DSF are larger than those of an SI-SMF for all working distances with the maximum efficiencies of $\sim 83 \%$ and $\sim 79 \%$ for the DSF and the SI-SMF, respectively. The longitudinal tolerance are of $\sim 60 \mu \mathrm{m}$ for a DSF and $\sim 40 \mu \mathrm{m}$ for an SI-SMF.

\section{Source-Waist Dependence}

The initial waist of the source seriously affects the characteristics of the proposed coupling scheme, as is clearly demonstrated in previous sections. In this section, we shall investigate the dependence of optimal coupling efficiency on source waists. The optimal coupling takes place when the incident beam curvature is compensated by the lens, ${ }^{12}$ and the transformed electric field can be expressed as $\mathbf{E}_{3}=$ $\exp \left(-r^{2} / W_{3}^{2}\right)$. The optimal beam waist for maximum overlapping with the $\mathrm{LP}_{02}$ cladding mode is $W_{3, \mathrm{opt}}=33.5 \mu \mathrm{m}$, which gives a coupling efficiency of $88 \%$. When the initial waist of the source $W_{0}$ and the optimal transformed field waist $W_{3, \text { opt }}$ are given, the optimal working distance $Z_{s}$ can be determined from Eq. (4) and the optimal lens radius $R_{f}$, from Eq. (5). In Fig. 9, we show the calculated optimal efficiencies versus the beam waists by use of the BPM and the $A B C D$ method. The corresponding optimal conditions of the lens radius and the working distance versus the beam waists by use of the BPM and the $A B C D$ method are shown in Fig. 10. Two measured coupling efficiencies and their optimal conditions corresponding to the LD-to-fiber coupling and the fiber-to-fiber coupling ${ }^{11,12}$ are also included in the figures. The optimal measured efficiency for the LD-to-fiber coupling is $35 \%$ for a $50-\mu \mathrm{m}$ lens radius and a $110-\mu \mathrm{m}$ working distance. For the fiber-tofiber coupling, the optimal measured efficiency is $78 \%$ for a $120-\mu \mathrm{m}$ lens radius and a $250-\mu \mathrm{m}$ working dis- 

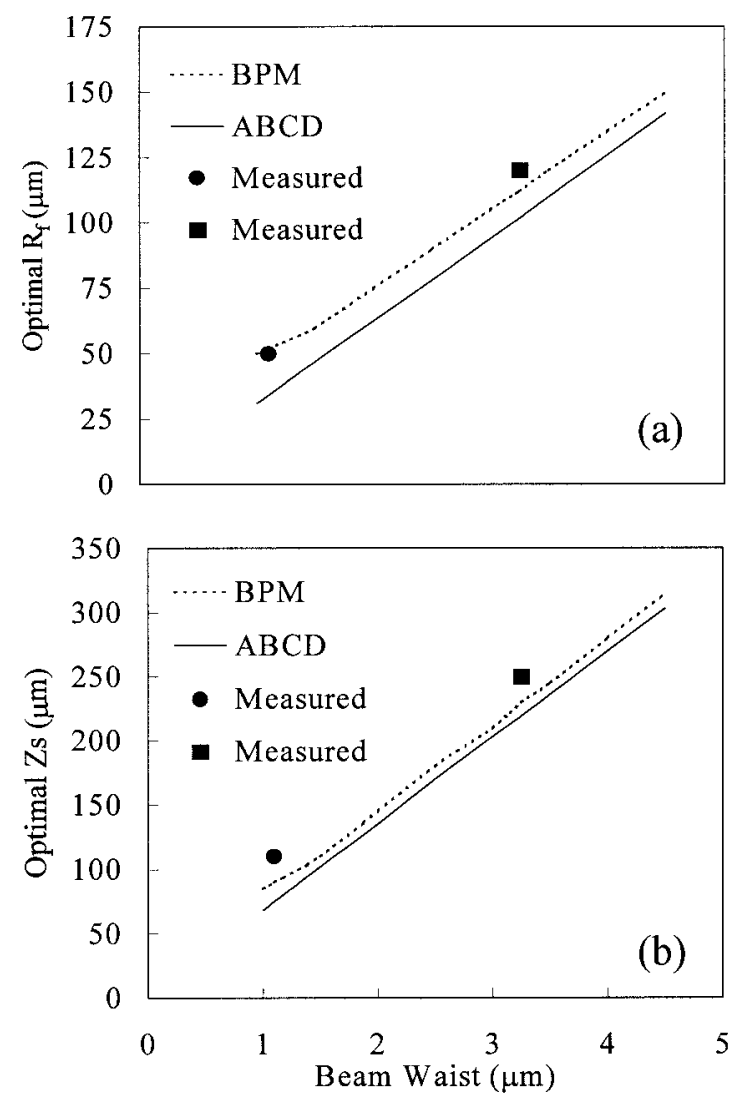

Fig. 10. Calculated and measured optimal conditions of (a) the lens radius $\left(R_{f}\right)$ and (b) the working distance $\left(Z_{s}\right)$ versus the beam waist. The measured one in the figures denoted as $(\bullet)$ is the case of LD-to-fiber coupling, and ( $\square$ ) is the case of fiber-to-fiber coupling.

tance. In Fig. 9, the optimal efficiency is a constant of $\sim 88 \%$ for beam waists from 1 to $4.5 \mu \mathrm{m}$, with the $A B C D$ method. However, the optimal coupling efficiency calculated from the BPM is only $40 \%$ for the source waist of $1 \mu \mathrm{m}$, and it monotonically increases with the waist and is saturated $\sim 87 \%$ for the waist beyond $4 \mu \mathrm{m}$. With consideration of the finite aperture of a lensed fiber and of beam truncation, the simulated results based on the BPM are more consistent with the measured ones.

\section{Conclusion}

The BPM has been applied to the analysis of beam evolution through the fiber lens in the coupling scheme reported previously. ${ }^{11,12}$ Since the effects of lens boundary and core cannot be properly accounted for in the $A B C D$ method, the BPM is considered a better approach for the analysis. We consider two kinds of waist sizes 1- $\mu \mathrm{m}$ for the small-waist source and $3.25-\mu \mathrm{m}$ for the large-waist source. From the BPM simulation, because of the large divergence angle, the incident beam from a small-waist source is truncated by the lens boundary, and partial energy is reflected from the interface. The reflected field then causes the oscillation of the coupling efficiency over the working distance. However, for the large-waist source, this effect is not significant. In addition, the existence of a fiber core has no significant effect on the coupling efficiency for the large- and the smallwaist sources. Note for the large-waist source, efficiencies can reach as much as $\sim 83 \%$ and $84 \%$ for the cored and coreless lenses, respectively. Also by our comparing the results from the BPM, the $A B C D$ method is found quite applicable to such analysis if the source has a large waist.

The calculated coupling efficiencies versus working distances are compared with the experiment results, and an overall agreement is obtained. As for the fiber dependence of our coupling scheme, the coupling constant $\kappa_{\text {co-cl }}$ of a DSF is larger than that of an SI-SMF, which explains why the transmission loss of the first cladding mode of an SI-SMF, is small. By studying the coupling behavior of two kinds of fibers, it was found that the longitudinal tolerance for a DSF is also larger. The dependence of optimal coupling efficiencies on source beam waists shows that the BPM is more consistent with the measured efficiencies and that the larger beam waist would lead to a better optimal coupling efficiency.

The authors thank W. T. Chen for helpful discussions and experiment data. The authors are grateful for the support in part by the National Science Council Taiwan, under the contract NSC 90-2215-E002-007 and by the Education Ministry under the contract 89-E-FA06-2-4 in Taiwan.

\section{References}

1. L. Cohen and M. Schneider, "Microlenses for coupling junction lasers to optical fibers," Appl. Opt. 13, 89-94 (1974).

2. J. Yamada, Y. Murakami, J. Sakai, and T. Kimura, "Characteristics of a hemispherical microlens for coupling between a semiconductor laser and single-mode fiber," IEEE J. Quantum Electron. QE-16, 1067-1072 (1980).

3. H. Kuwahara, M. Sasaki, and N. Tokoyo, "Efficient coupling from semiconductor lasers into single-mode fibers with tapered hemispherical ends," Appl. Opt. 19, 2578-2583 (1980).

4. K. S. Lee and F. S. Barnes, "Microlenses on the end of singlemode optical fibers for laser applications," Appl. Opt. 24, 31343139 (1985).

5. K. Shiraishi, "New scheme of coupling from laser diodes to single-mode fibers: a beam expanding fiber with a hemispherical end," Opt. Lett. 29, 3467-3469 (1990).

6. C. A. Edwards, H. M. Presby, and C. Dragone, "Ideal microlenses for laser to fiber coupling," J. Lightwave Technol. 11, 252-257 (1993).

7. W. Bludau and R. Rossberg, "Characterization of laser-to-fiber coupling techniques by their optical feedback," Appl. Opt. 21, 1933-1939 (1982).

8. K. Shiraishi, N. Oyama, K. Matsumura, I. Ohishi, and S. Suga, "A fiber lens with a long working distance for integrated coupling between laser diodes and single-mode fibers," J. Lightwave Technol. 13, 1736-1744 (1995).

9. K. Shiraishi, H. Ohnuki, N. Hiraguri, K. Matsumura, I. Ohishi, H. Morichi, and H. Kazami, "A lensed-fiber coupling scheme utilizing a graded-index fiber and a hemispherically ended coreless fiber tip," J. Lightwave Technol. 15, 356-363 (1997).

10. K. Shiraishi and S.-I. Kuroo, "A new lensed-fiber configuration employing cascaded GI-fiber chips,” J. Lightwave Technol. 18, 787-794 (2000).

11. W. T. Chen and L. A. Wang, "Laser-to-fiber coupling scheme by 
utilizing a lensed fiber integrated with a long-period fiber grating," IEEE Photon. Technol. Lett. 12, 501-503 (2000).

12. W. T. Chen and L. A. Wang, "Optical coupling method utilizing a lensed fiber integrated with a long-period fiber grating," Appl. Opt. 39, 4490-4500 (2000).

13. A. M. Vengsarkar, P. J. Lemaire, J. B. Judkins, V. Bhatia, T. Erdogan, and J. Sipe, "Long-period fiber gratings as bandrejection filters," J. Lightwave Technol. 4, 58-65 (1996).

14. T. Erdogan, "Cladding-mode resonances in short- and longperiod fiber grating filters," J. Opt. Soc. Am. A 14, 1760-1773 (1997).

15. M. D. Feit and J. A. Fleck, Jr., "Light propagation in gradedindex optical fibers," Appl. Opt. 17, 3990-3998 (1978).

16. B. Hermansson, D. Yevick, and J. Saijonmaa, "Propagationbeam-method analysis of two-dimensional microlenses and three-dimensional taper structures," J. Opt. Soc. Am. A 1, 663-671 (1984)

17. F. Gonthier, A. Henault, S. Lacroix, R. J. Black, and J. Bures, "Mode coupling in nonuniform fibers: comparison between coupled-mode theory and finite-difference beam-propagation method simulations," J. Opt. Soc. Am. B 8, 416-421 (1991).

18. J. P. Zhang and K. Petermann, "Beam propagation model for vertical-cavity surface-emitting lasers: threshold properties," IEEE J. Quantum. Electron. 30, 1529-1536 (1994).

19. J. Yamauchi, Y. Akimoto, M. Nibe, and H. Nakano, "Wideangle propagation analysis for circularly symmetric waveguides: comparison between FD-BPM and FD-TDM," IEEE Photon. Technol. Lett. 8, 236-238 (1996).

20. G. R. Hadley, "Transparent boundary condition for beam propagation," Opt. Lett. 16, 624-626 (1991). 